\title{
The MicroRNA-210/Casp8ap2 Pathway Alleviates Hypoxia-Induced Injury in Myocardial Cells by Regulating Apoptosis and Autophagy
}

\author{
Kunsheng Li, ${ }^{1,2, *}$ Jun Pan, ${ }^{1, *}$ Qiuchang Li, ${ }^{3}$ Shiliang Li, ${ }^{6}$ Kai Li, ${ }^{1}$ Yongqing Cheng, ${ }^{1}$ Lin Chai, ${ }^{1}$ Chao Li, \\ Junling Li, ${ }^{3}$ Zhikun Fu, ${ }^{5}$ Dongjin Wang, ${ }^{1, \#}$ Yang Bai ${ }^{7, \#}$ \\ ${ }^{1}$ Department of Cardiothoracic Surgery, Nanjing Drum Tower Hospital, The Affiliated Hospital of Nanjing University Medical \\ School, Jiangsu Province; ${ }^{2}$ Department of Cardiac Surgery, University of Heidelberg, Heidelberg, Germany; ${ }^{3}$ Puyang Medical \\ College, Shangyang Road and Wenyan Street, Puyang, Henan Province; ${ }^{4}$ School of Life Sciences, Anhui Medical University, \\ Anhui Province; ${ }^{5}$ Department of Cardiac Surgery, The 7th People's Hospital of Zhengzhou, Henan Province; ${ }^{6}$ Department of \\ Cardiac Surgery, Tongji Hospital, Tongji Medical College, Huazhong University of Science and Technology, Wuhan, Hubei \\ Province; ${ }^{7}$ Division of Cardiology, Department of Internal Medicine, Tongji Hospital, Tongji Medical College, Huazhong \\ University of Science and Technology, Wuhan, Hubei Province, P.R. China
}

\section{ABSTRACT}

Aim: This study was conducted to investigate the role of the miR-210/Caspase8ap2 pathway in apoptosis and autophagy in hypoxic myocardial cells.

Methods: The miR-control, miR-210 mimic, and miR-210 inhibitor were transfected into rat myocardial H9C2 cells. The transfection efficiency of exogenous miR-210 was determined by quantitative reverse-transcription polymerase chain reaction (qRT-PCR). H9C2 cells were then treated with $\mathrm{CoCl}_{2}$ for 24, 48 , and $72 \mathrm{~h}$ to generate a myocardial injury model. The apoptosis of H9C2 cells was assessed by flow cytometry. Additionally, a western blot assay was used to determine the expression of the autophagy-associated proteins light chain 3 (LC3), p62 and Beclin-1, and apoptosis-associated proteins Caspase8ap2, cleaved caspase 8 , and cleaved caspase 3 .

Results: We determined that a $48 \mathrm{~h}$ hypoxia treatment duration in $\mathrm{H} 9 \mathrm{C} 2$ cardiomyocytes induced myocardial injury. Additionally, the overexpression of miR-210 significantly inhibited cell apoptosis. MiR-210 suppressed autophagy by upregulating p62 and downregulating LC3II/I in hypoxic H9C2 cells. Caspase8ap2 was a putative target of miR-210, miR-210 mediated apoptosis, and autophagy of H9C2 cells via suppressing Caspase8ap2. Furthermore, the expression of caspase 8, caspase 3, and Beclin-1 were decreased in response to miR-210.

Received Fuly 6, 2020; received in revised form August 22, 2020; accepted August 24, 2020.

*Both authors contributed equally to this work.

This study was supported by The National Key Clinical Speciality Construction Project of China (ZXA201101).

\#Correspondence: Dr. Yang Bai, Division of Cardiology, Department of Internal Medicine, Tongji Hospital, Tongji Medical College, Huazhong University of Science and Technology, Fiefang Road, No 1095, Wuban 430030, Hubei Province, P.R. China; (email: liam.li_de@foxmail.com) Co-correspondence: Professor Dongjin Wang, (email: dongjin.wang_nj@outlook.com).
Conclusion: miR-210 exhibits anti-apoptosis and antiautophagy effects, which alleviate myocardial injury in response to hypoxia.

\section{INTRODUCTION}

Coronary heart disease (CHD) is characterized by myocardial ischemia, anoxia, and necrosis attributed to coronary atherosclerosis, which results in heart defects. CHD is a leading cause of death and disability, with a 10 -year mortality rise of 60\% in China [Greenwood 2012; Rastam 2012]. This devastating rise is mainly due to adverse trends in risk factors, such as blood pressure, cholesterol, and diabetes [Heran 2011]. Identifying mechanisms to inhibit myocardial cell apoptosis under a hypoxic-ischemic state is a recent topic of high interest in CHD research.

Many studies have shown that microRNAs (miRNAs), an abundant class of endogenic non-coding RNAs with approximately 22 nucleotides, are involved in a wide variety of biological processes by modulating the expression of target genes and pathways [Muhammad 2016; Shimono 2015]. For CHD, an increasing number of miRNAs have been identified that play considerable roles in CHD development, such
A

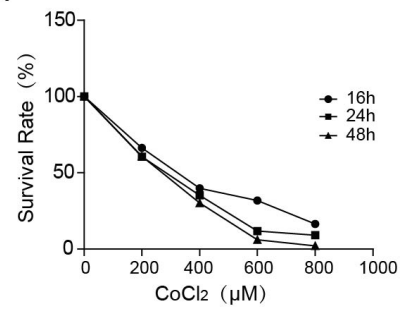

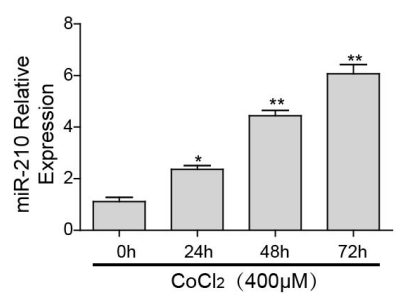

Figure 1. Effects of $\mathrm{CoCl}_{2}$ on hypoxic myocardial cells. A, Effects of $\mathrm{CoCl}_{2}$ concentration and treatment duration on the apoptotic ratio of H9C2 cells; B, Effects of different hypoxic treatment times on the relative miR-210 expression. 
A

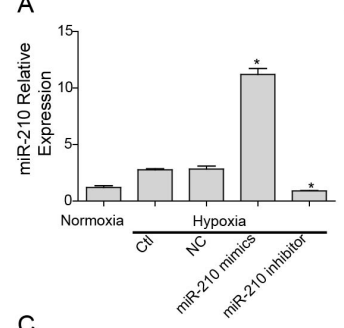

B

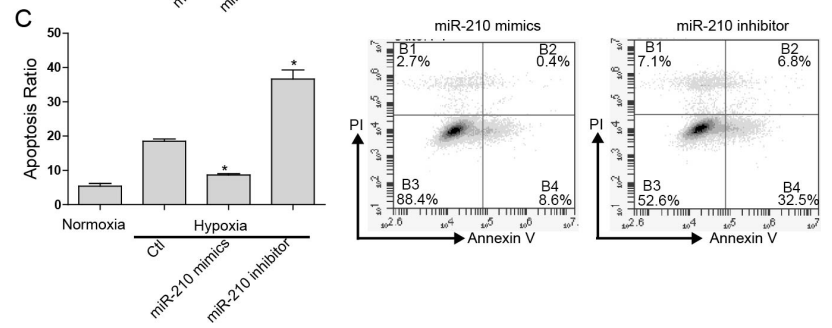

Figure 2. Effects of miR-210 on cell apoptosis in hypoxic cardiomyocytes. A, Transfection efficiency in NC, miR-210 mimic, and miR-210 inhibitor groups; B, Effects of miR-210 mimic and miR-210 inhibitor groups on $\mathrm{H} 9 \mathrm{C} 2$ cell apoptosis; $\mathrm{C}, \mathrm{H} 9 \mathrm{C} 2$ cell apoptosis was analyzed by flow cytometry.

as miR-320b [Feng 2014], miR-224 [Miller 2014], miR-423, miR-208a, and miR-1 [Nabialek 2013].

Among various miRNAs, miR-210 plays a significant role in the hypoxia response on the account of its high sensitivity to hypoxia. Recent evidence has emerged that suggests miR-210, as the main hypoxia-induced miRNA, has pivotal implications in cell survival and angiogenesis [Huang 2009]. During hypoxia stress, miR-210 was potently upregulated in myocardial cells in vitro [Mutharasan 2011]. In addition, targeted miR-210 could effectively protect the heart from myocardial infarctions and promote functional recovery $[\mathrm{Hu}$ 2010]. However, little is known about the mechanism of miR210 in ischemia-hypoxia in myocardial cells.

Caspase-8-associated protein-2 (Caspase8ap2) consists of 1926 amino acids and is an integral member of the apoptosis signalling complex that activates caspase- 8 and downstream caspase 3 by combining with the death receptor of procaspase-8 [Kim 2009]. Thus, Caspase8ap2 is considered a proapoptosis protein. Kim et al reported that the apoptosis in mesenchymal stem cells (MSCs) caused by ischemic preconditioning (IP) was suppressed by miR-210 by repressing Caspase8ap2 [Kim 2009].

The present study was conducted to investigate the role of the miR-210/Caspase8ap2 pathway in apoptosis and autophagy in hypoxic myocardial cells. The findings of this study can potentially provide novel avenues for the treatment of myocardial injury induced by hypoxia.

\section{MATERIALS AND METHODS}

\section{Hypoxic Myocardial Injury Model}

The rat myocardial cell line H9C2 (American Type Culture Collection, Manassas, VA, USA) was cultured in Dulbecco's
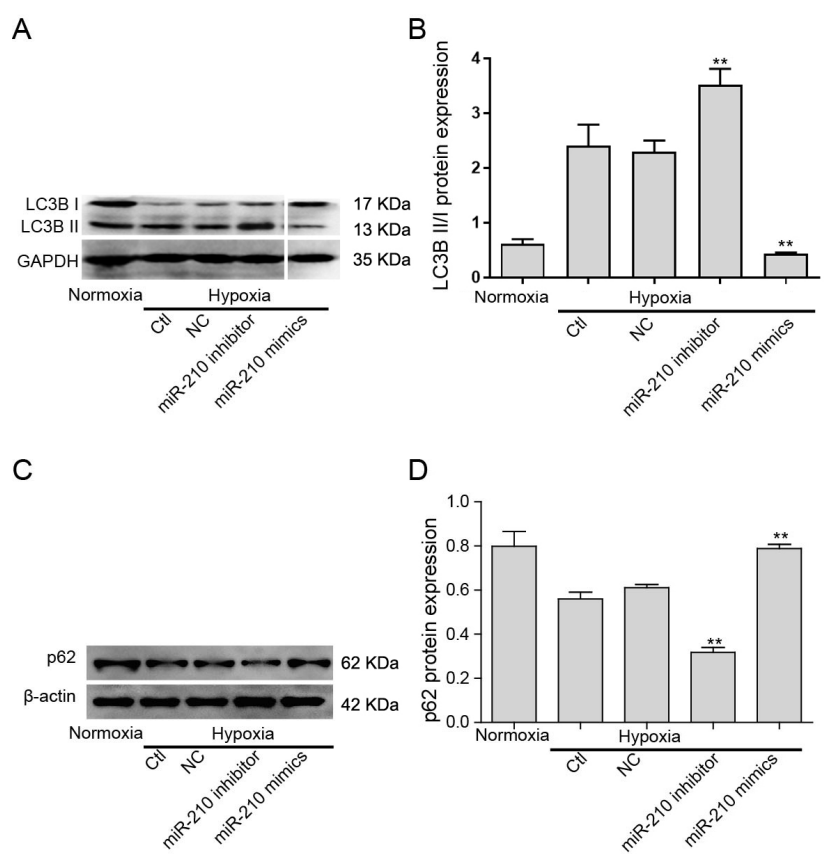

Figure 3. Effects of miR-210 on autophagy in hypoxic cardiomyocytes. A, Expression of LC3I and LC3II proteins in the five groups; B, The grey value analysis of LC3 II/I expression in $\mathrm{H} 9 \mathrm{C} 2$ cells; C, Expression of $\mathrm{p} 62$ proteins in $\mathrm{H} 9 \mathrm{C} 2$ cells; $D$, The grey value analysis of p62 (of $\beta$-actin) in the five groups. LC3 indicates light chain 3; NC, negative control.

Modified Eagle's Medium (DMEM, Gibco-BRL, Grand Island, NE, USA) supplemented with $10 \%$ fetal bovine serum (FBS; HyClone, Logan, USA). All cells were maintained in a humidified incubator containing 95\% air and 5\% CO2 at $37^{\circ} \mathrm{C}$. Cells were cultured to $70-80 \%$ confluence, which was determined using a phase contrast microscopy (Olympus, Tokyo, Japan). After reaching $70-80 \%$ confluence, H9C2 cells were treated with $400 \mu \mathrm{M} \mathrm{CoCl}$, for 24,48 , and $72 \mathrm{~h}$.

\section{Cell Transfection}

Hypoxic H9C2 cells were seeded at a density of $3 \times 104$ cells/well in six-well plates and allowed to adhere to the dish overnight. The next day, the miR-negative control (NC), miR-210 mimic, and miR-210 inhibitor (GenePharma Co, Shanghai, China) were transfected into H9C2 cells using Lipofectamine 2000 reagent (Life Technologies, Gaithersburg, MD, USA) according to manufacturer's protocol.

\section{Quantitative Reverse-Transcription Polymerase Chain Reaction (qRT-PCR)}

Total RNA was extracted from normal H9C2 cells, hypoxic H9C2 cells, NC, miR-210 mimic, and miR-210 inhibitor group with TRIzol reagent (Invitrogen, Carlsbad, CA, USA) following the manufacturer's instructions. Briefly, singlestranded complementary DNA (cDNA) was synthesized from $2 \mu \mathrm{g}$ of RNA via the RevertAid First Strand cDNA Synthesis Kit (Fermentas, Ontario, Canada). Subsequently, the generated miR-210 cDNA was amplified via qRT-PCR based on the TaqMan microRNA assay protocol (Roche LightCycler 
A

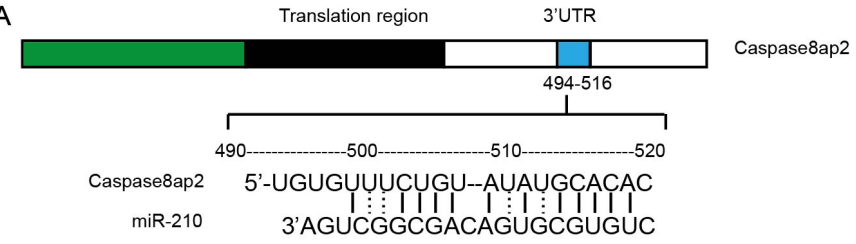

B

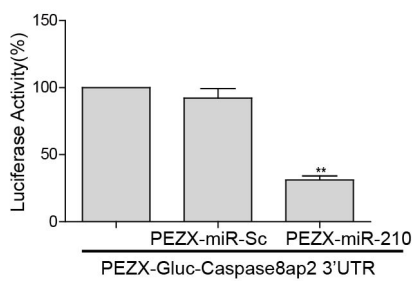

Figure 4. Caspase8ap2 is a putative target of miR-210 in $\mathrm{H} 9 \mathrm{C} 2$ cells. A, The putative target site of miR-210 was highly conserved in the Caspase8ap2 mRNA 3'UTR; B, Caspase8ap2 3'UTR along with a plasmid encoding miR-210; ${ }^{* * P}<.01$.

480 II System). Briefly, $20 \mu \mathrm{L}$ reactions were incubated in a 96-well optical plate at $94^{\circ} \mathrm{C}$ for $2 \mathrm{~min}$ and then subjected to 40 cycles of $94^{\circ} \mathrm{C}$ for $20 \mathrm{~s}$ and $60^{\circ} \mathrm{C}$ for $34 \mathrm{~s}$. The relative expression of miR-210 was normalized to the internal control (U6) using the $2^{-\Delta \Delta \mathrm{Ct}}$ method. The primers for miR-210 and U6 were synthesized with the miScript Primer Assay kit (Qiagen, Dusseldorf, Germany).

\section{Apoptosis Assay}

Apoptotic cells in NC, miR-210 mimic, and miR-210 inhibitor groups were quantified using the Annexin V-FITC apoptosis detection kit (Beyotime Institute of Biotechnology, Jiangsu, China). Both the attached cells and cells in the supernatant were collected from the above four groups and fixed with $2 \%$ paraformaldehyde. They were washed twice with cold phosphate-buffered saline (PBS) and resuspended in $100 \mu \mathrm{L}$ of the annexin-binding buffer. Then, H9C2 cells were incubated with $2.5 \mu \mathrm{L}$ of Annexin $\mathrm{V}$ for $15 \mathrm{~min}$ and analyzed using an EPICS XL flow cytometer (Beckman-Coulter, Fullerton, CA, USA). Apoptotic cells were defined as the cells that belonged to the right quadrant (right top + right bottom) in flow cytometry plots. The experiment was independently repeated three times, and an average apoptotic rate was calculated.

\section{Western Blot}

Standard western blotting was conducted for protein expression assays in hypoxic H9C2 cells transfected with miR-210 mimic, inhibitor, and NC. Two days following transfection, proteins were isolated with RIPA lysis buffer containing $1 \mathrm{mg}$ protease inhibitors (Applygen Technologies, Beijing, China). The protein contents were quantified using the Bicinchoninic Acid (BCA) Protein Assay Kit (CoWin Biotech, Beijing, China). Primary antibodies against light chain 3 (LC3; ab48394), p62 (ab56416), Caspase8ap2 (ab4052), cleaved caspase 8 (ab25901), cleaved caspase 3 (ab13847), Beclin-1 (ab62557), and the internal controls
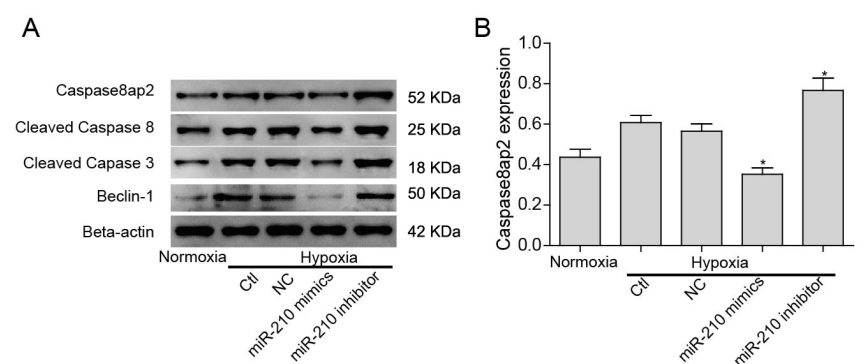

C

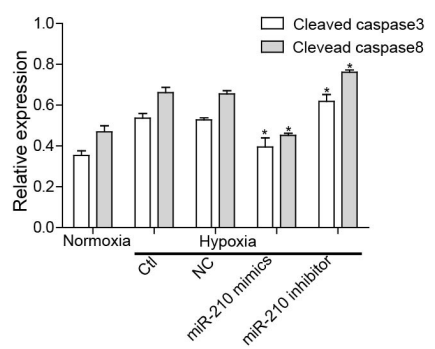

$\mathrm{D}$

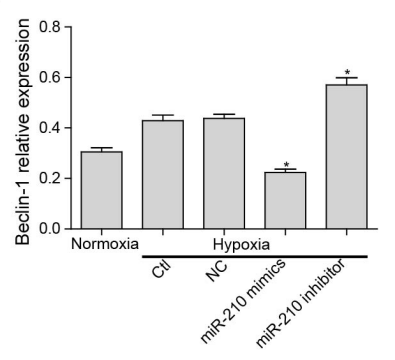

Figure 5. Effects of miR-210 on Caspase8ap2 signalling pathway. A, Expression of Caspase8ap2, caspase 8, caspase 3, and Beclin-1 proteins determined by western blot; B, Effects of exogenous miR-210 expression on the protein level of Caspase8ap2; C, Effects of miR-210 overexpression on caspase 8 and caspase 3 protein levels; D, Effects of exogenous miR-210 on the protein level of Beclin-1; NC indicates negative control.

-actin and GAPDH were from Abcam (Cambridge, UK). Furthermore, secondary antibodies labelled with horseradish peroxidase were incubated with membranes for $1 \mathrm{~h}$ at $37^{\circ} \mathrm{C}$. Samples were then transferred to a polyvinylidene fluoride (PVDF) microporous membrane (Millipore, Boston, MA, USA). Blots were imaged by a FluorChem E imager (Cell Biosciences, San Jose, CA, USA). The grey value of each band was determined with ImageJ version 1.51.

\section{Luciferase Reporter Assay}

For transfections, host H9C2 cells were plated in triplicate into 24-well plates containing Lipofectamine 2000 (Invitrogen, Carlsbad, CA, USA) and $0.8 \mu \mathrm{g}$ pEZX-miR-210 (or pEZX-miR-Sc) per well. The precursor miR-210 expression clone was established via a feline immunodeficiency virus using the lentiviral vector system (pEZX-miR-210). Subsequently, we designed the luciferase reporter targeting the 3-UTR of Caspase8ap2 to surround the binding sites of miR-210. The Dual-Luciferase Reporter Assay System kit (Promega, Madison, WI, USA) was used to measure the amount of luciferase and Renilla luciferase activity with a fluorescence spectrophotometer. The relative transcriptional activity was normalized to its corresponding value.

\section{Statistical Analysis}

All data were expressed as the mean \pm standard deviation (Claes, \#329). Statistical differences were evaluated by SPSS 19.0 software. Statistical analysis was performed using a oneway analysis of variance and Student $t$ test, and a $P$ value $<.05$ was considered statistically significant. 


\section{RESULTS}

\section{Expression of miR-210 in H9C2 was Upregulated in Hypoxic H9C2 Cells}

To generate an optimized model of anoxic myocardial cells, H9C2 cells were treated with $\mathrm{CoCl}_{2}$ for 24,48 , and $72 \mathrm{~h}$. The number of apoptotic cells and relative miR-210 expression are shown in Figure 1. With increasing $\mathrm{CoCl}_{\text {, concentrations }}$ and treatment duration, the apoptosis ratio of $\mathrm{H} 9 \mathrm{C} 2$ cells was enhanced (Figure 1, A). Additionally, the relative expression of miR-210 increased in a time-dependent manner (Figure $1, \mathrm{~B})$. We found that a $24 \mathrm{~h}$ and $48 \mathrm{~h}$ hypoxia treatment provoked similar apoptosis ratios in $\mathrm{H} 9 \mathrm{C} 2$ cells. Therefore, a 48 h hypoxia duration was used in $\mathrm{H} 9 \mathrm{C} 2$ cardiomyocytes for all subsequent experiments.

\section{Overexpressing miR-210 Inbibited Cell Apoptosis in Hypoxic H9C2 Cells}

We evaluated the effects of miR-210 overexpression on cell apoptosis in hypoxic cardiomyocytes by generating $\mathrm{NC}$, miR-210 mimic, and miR-210 inhibitor groups under hypoxia culture for $48 \mathrm{~h}$. The relative expression of miR-210 was potently enhanced in the miR-210 mimic group but was suppressed in the miR-210 inhibitor group, which demonstrated the high transfection efficiency in H9C2 cells (Figure 2, A). Moreover, there was no significant difference in the apoptosis ratio between hypoxia and NC groups (data not shown). Overexpressing miR-210 caused a decline in apoptosis, whereas silencing miR-210 had the opposite effect. It was uncovered that overexpressing miR-210 inhibited cell apoptosis in hypoxic H9C2 cells (Figure 2, B and C).

\section{Overexpressing miR-210 Inbibited Autophagy in Hypoxic H9C2 Cells}

Next, the expression of autophagy-associated proteins LC3 and $\mathrm{p} 62$ were assessed by western blot to investigate the effects of miR-210 overexpression on autophagy in hypoxic H9C2 cells. As shown in Figure 3, LC3 II/I was upregulated and p62 was downregulated in the hypoxia group compared with the normoxia group, which demonstrated that there was a certain degree of autophagy in H9C2 cells. Furthermore, the expression of LC3 II/I was significantly lower, and the expression of p62 was higher in the miR-210 mimic group compared with other groups, which indicated that miR-210 overexpression inhibited autophagy in $\mathrm{H} 9 \mathrm{C} 2$ cells under hypoxic conditions.

\section{Caspase8ap2 Was a Target Gene of miR-210}

To explore whether Caspase8ap2 was a target gene of miR210 , we performed a dual-luciferase reporter gene assay in H9C2 cells. Our computational studies identified consensus putative target sites of miR-210 with high complementarity relevant to the 3'UTR region of Caspase8ap2 (Figure 4, A). For the luciferase reporter assay, a dual-luciferase construct was generated. Remarkably decreased luciferase activity was observed on pEZX-miR-201 co-transfecting with the pEZXLuc-Caspase8ap2 3'UTR (Figure 4, B; $P<.01$ ). These results showed that miR-210 could inhibit the activity of luciferase on the Caspase8ap2 3'UTR reporter gene cattier.
miR-210 Mediated Apoptosis and Autophagy of H9C2 Cells via Targeted-Regulating of Caspase8ap2

To further investigate the mechanism of miR-210 in regulating Caspase8ap2 and its associated signalling pathway, we determined the effects of exogenous miR-210 on the expression of Caspase8ap2 and downstream associated proteins by western blot (Figure 5, A). Beclin-1 was upregulated in the hypoxia group compared with the normoxia group. Protein levels of Caspase8ap2, cleaved caspase 8, and cleaved caspase 3 were decreased in response to overexpressing miR-210 (Figure 5, B and C). Beclin-1, which regulates both apoptosis and autophagy, was also downregulated in the miR-210 mimic group (Figure 5, D). On the other hand, the expression of Caspase8ap2 and other proteins in response to silencing miR-210 were increased. Therefore, miR-210 likely mediates apoptosis and autophagy in $\mathrm{H} 9 \mathrm{C} 2$ cells via targetedregulation of Caspase8ap2.

\section{DISCUSSION}

Myocardial hypoxia is a negative prognostic marker associated with myocardial injury and a poor patient outcome. It is also a primary component of CHD microenvironment [Kuo 2016]. Recent work supports the idea that miRNAs are involved in the adaptive response to myocardial hypoxia in cardiovascular diseases. Liu et al suggested that miR-130a participated in hypoxia/reoxygenation (H/R) induced injuries by accelerating autophagy and relieving apoptosis in cardiomyocytes [Liu 2017]. Moreover, the silencing of miR-122 alleviated cardiomyocyte H/R injury by promoting GATA-4 expression [Liang 2016]. Li et al reported that miR-7a/b protected $\mathrm{H} 9 \mathrm{C} 2$ cells from hypoxia-induced injury and promoted cardiac remodelling by decreasing fibrosis and apoptosis [Li 2016]. In particular, for the hypoxic response, all published literature agrees that miR-210 is a robust target of hypoxia-inducible factors. It is well known that the upregulation of miR-210 is a hypoxic signature in vivo [Huang 2014]. Hence, miR-210-mediated myocardial hypoxia may represent an attractive target for therapeutic intervention in CHD. However, the mechanisms involved in miR-210 repair of myocardial hypoxia remains to be elucidated. Our study was performed to identify the role of miR-210 in a hypoxic $\mathrm{H} 9 \mathrm{C} 2$ model, which might contribute to the development of potential diagnostic and therapeutic approaches in CHD. First, we determined that a $48 \mathrm{~h}$ hypoxia treatment duration in $\mathrm{H} 9 \mathrm{C} 2$ cardiomyocytes induced myocardial injury. Also, miR-210 significantly inhibited cell apoptosis and autophagy in hypoxic H9C2 cells. Since Caspase8ap2 was a putative target of miR210, miR-210 likely mediated apoptosis and autophagy of H9C2 cells via suppressing Caspase8ap2. Taken together, the miR-210/Caspase8ap2 pathway alleviated the effects of $\mathrm{CoCl}_{2}$ induced injury in $\mathrm{H} 9 \mathrm{C} 2$ cells by regulating apoptosis and autophagy. There are few published articles on the effects of miR-210 on the other two apoptotic signaling pathways in cardiomyocytes. Besides death receptor/Caspase8 pathway, mitochondria/Caspase9 and ER stress/Caspase12 pathways are also major signalling pathways in apoptosis $[\mathrm{He} 2016 ; \mathrm{Pu}$ 
2013]. Further studies on the association between miR-210 and other potential target apoptotic genes in cardiomyocytes with hypoxia need to be conducted.

In the miRNA field, extensive evidence consistently points to a dominant role for miR-210 in the hypoxic stress response [Huang 2010]. The induction of apoptosis in cells subjected to hypoxia has been of great importance to cell survival and tissue homeostasis. Numerous previous reports have supported an anti-apoptotic role of miR-210 during hypoxia. For example, miR-210 exhibited cytoprotective effects from apoptosis [Mutharasan 2011; Nie 2011, while the downregulation of miR-210 induced apoptosis [Yang 2012; Gou 2012]. Similar results were verified in this study in which the overexpression of miR-210 inhibited apoptosis, whereas silencing miR-210 strikingly enhanced apoptosis in hypoxic H9C2 cells. Further, Caspase8ap2 has been predicted as a downstream target of miR-210 [Kim 2012]. Caspase8ap2 was initially identified as a member of the death signalling complex joining Fas and tumor necrosis factor- (TNF-) during apoptosis [Kim 2009]. Its interaction with death effectors promotes the delivery of caspase- 8 from the apoptosis pathway to activate the downstream caspase cascade. Because of its role in apoptosis, we validated Caspase8ap2 for its functional involvement in $\mathrm{H} 9 \mathrm{C} 2$ apoptosis under hypoxic conditions using the dual-luciferase reporter assay. Western blot analysis demonstrated that miR-210 overexpression reduced the protein level of Caspase8ap2, cleaved caspase 8, and caspase 3 upon exposure to hypoxia, suggesting that miR-210 inhibited cardiomyocyte apoptosis by modulating the levels of Caspase8ap2.

The double-edged sword biological function of autophagy in myocardial hypoxia injury was demonstrated by recent studies. Moderate autophagy contributes to ATP generation and cellular homeostasis when cells are exposed to nonserious injury. However, uncontrolled excessive autophagy could be induced by serious or long-term injury. Excessive autophagy may contribute to autophagic cardiomyocyte death [Ma 2015; Hamacher-Brady 2006]. In our study, $\mathrm{CoCl}_{2}$ treatment for $48 \mathrm{~h}$ significantly inhibited cell survival. Hence, under this serious injury condition, we assumed that excessive autophagy was induced, and it may have contributed to cell death. Autophagy and apoptosis are considered to be highly interconnected through mechanisms that involve Beclin-1 [Joubert 2012]. Beclin-1 is necessary for autophagy to occur, can direct autophagy-related proteins to phagocytes, and can regulate autophagy formation and maturation with other proteins. Although it is widely accepted that autophagy can promote survival in response to milder stress, excessive and/or long-term autophagy can also cause cell death by promoting the excessive self-digestion of essential organelles and proteins. In the current study, both the upregulation of adapter protein $\mathrm{p} 62$ and downregulation of autophagy indicators LC3 II/I and Beclin-1 showed that miR-210 may protect H9C2 cells from excessive autophagy in response to hypoxia.

In conclusion, miR-210 collectively exerts antiapoptosis and anti-autophagy effects, which alleviates myocardial injury in response to hypoxia. This study suggests that the miR-210/Caspase8ap2 pathway is essential to protect cells from hypoxia. Future studies aimed at further characterizing the underlying mechanism of miR-210 in other apoptosisassociated pathways should be conducted.

\section{We would like to give our sincere gratitude to the reviewers for their belpful comments on this paper.}

\section{REFERENCES}

Feng J, Huang S, He M, et al. 2014. Analysis on the association of single nucleotide polymorphism in the promoter region of pre-miR-320b-2 with coronary heart disease risk and factors influencing circulating microRNA-320b level. Zhonghua Yu Fang Yi Xue Za Zhi. 48:893-9.

Gou D, Ramchandran R, Peng X, et al. 2012. miR-210 has an antiapoptotic effect in pulmonary artery smooth muscle cells during hypoxia. Am J Physiol Lung Cell Mol Physiol 303: L682-91.

Greenwood JP, Maredia N, Younger JF, et al. 2012. Cardiovascular magnetic resonance and single-photon emission computed tomography for diagnosis of coronary heart disease (CE-MARC): a prospective trial. Lancet 379:453-60.

Hamacher-Brady A, Brady NR,Gottlieb RA. 2006. The interplay between pro-death and pro-survival signaling pathways in myocardial ischemia/reperfusion injury: apoptosis meets autophagy. Cardiovasc Drugs and Therapy 20:445-62.

He B, Zhao Y, Xu L, et al. 2016. The nuclear melatonin receptor RORalpha is a novel endogenous defender against myocardial ischemia/reperfusion injury. J Pineal Res 60:313-26.

Heran BS, Chen JM, Ebrahim S, et al. 2011. Exercise-based cardiac rehabilitation for coronary heart disease. Cochrane Database Syst Rev 7:CD001800.

Hu S, Huang M, Li Z, et al. 2010. MicroRNA-210 as a novel therapy for treatment of ischemic heart disease. Circulation 122(11 Suppl):S124-31.

Huang X, Ding L, Bennewith KL, et al. 2009. Hypoxia-inducible mir210 regulates normoxic gene expression involved in tumor initiation. Mol Cell 35:856-67.

Huang X, Le QT, Giaccia AJ. 2010. MiR-210--micromanager of the hypoxia pathway. Trends Mol Med 16:230-7.

Huang X, Zuo J. 2014. Emerging roles of miR-210 and other non-coding RNAs in the hypoxic response. Acta Biochim Biophys Sin (Shanghai). 46:220-32.

Joubert PE, Werneke SW, de la Calle C, et al. 2012. Chikungunya virusinduced autophagy delays caspase-dependent cell death. J Exp Med 209:1029-47.

Kim HW, Haider HK, Jiang S, Ashraf M. 2009. Ischemic preconditioning augments survival of stem cells via miR-210 expression by targeting caspase-8-associated protein 2. J Biol Chem 284:33161-8.

Kim HW, Mallick F, Durrani S, Ashraf M, Jiang S, Haider KH. 2012. Concomitant activation of miR-107/PDCD10 and hypoxamir-210/Casp8ap2 and their role in cytoprotection during ischemic preconditioning of stem cells. Antioxid Redox Signal 17:1053-65.

Kuo HF, Liu PL, Chong IW, et al. 2016. Pigment epithelium-derived factor mediates autophagy and apoptosis in myocardial hypoxia/reoxygenation injury. PLoS One 11:e0156059.

Li R, Geng HH, Xiao J, et al. 2016. miR-7a/b attenuates post-myocardial infarction remodeling and protects $\mathrm{H} 9 \mathrm{c} 2$ cardiomyoblast against 
hypoxia-induced apoptosis involving Sp1 and PARP-1. Sci Rep 6:29082.

Liang W, Guo J, Li J, Bai C, Dong Y. 2016. Downregulation of miR122 attenuates hypoxia/reoxygenation (H/R)-induced myocardial cell apoptosis by upregulating GATA-4. Biochem Biophys Res Commun. 478:1416-22.

Liu H, Huan L, Yin J, et al. 2017. Role of microRNA-130a in myocardial hypoxia/reoxygenation injury. Exp Ther Med 13:759-65.

Ma S, Wang Y, Chen Y,Cao F. 2015. The role of the autophagy in myocardial ischemia/reperfusion injury. Biochimica et biophysica acta 1852:271-6.

Miller CL, Haas U, Diaz R, et al. 2014. Coronary heart disease-associated variation in TCF2 1 disrupts a miR-224 binding site and miRNAmediated regulation. PLoS Genet 10:e1004263.

Muhammad N, Bhattacharya S, Steele R, Ray RB. 2016. Anti-miR-203 suppresses ER-positive breast cancer growth and stemness by targeting SOCS3. Oncotarget 7:58595-605.

Mutharasan RK, Nagpal V, Ichikawa Y, Ardehali H. 2011. microRNA-210 is upregulated in hypoxic cardiomyocytes through Akt- and p53-dependent pathways and exerts cytoprotective effects. Am J Physiol Heart Circ
Physiol 301:H1519-30.

Nabialek E, Wanha W, Kula D, et al. 2013. Circulating microRNAs (miR-423-5p, miR-208a and miR-1) in acute myocardial infarction and stable coronary heart disease. Minerva Cardioangiol 61:627-37.

Nie Y, Han BM, Liu XB, et al. 2011. Identification of MicroRNAs involved in hypoxia- and serum deprivation-induced apoptosis in mesenchymal stem cells. Int J Biol Sci 7:762-8.

$\mathrm{Pu}$ J, Yuan A, Shan P, et al. 2013. Cardiomyocyte-expressed farnesoid$\mathrm{X}$-receptor is a novel apoptosis mediator and contributes to myocardial ischaemia/reperfusion injury. Eur Heart J 34:1834-45.

Rastam S, Al Ali R, Maziak W, et al. 2012. Explaining the increase in coronary heart disease mortality in Syria between 1996 and 2006. BMC Public Health 12:754.

Shimono Y, Mukohyama J, Nakamura S, Minami H. 2015. MicroRNA regulation of human breast cancer stem cells. J Clin Med 5:pii:E2.

Yang W, Sun T, Cao J, Liu F, Tian Y, Zhu W. 2012. Downregulation of miR-210 expression inhibits proliferation, induces apoptosis and enhances radiosensitivity in hypoxic human hepatoma cells in vitro. Exp Cell Res 318:944-54. 